\title{
Comparative Study Evaluating Lumen Apposing Metal Stents Versus Double Pigtail Plastic Stents for Treatment of Walled Off Necrosis
}

\author{
Phillip S. Ge, MD ${ }^{* 1,2}$, Joyce Y. Young, JD ${ }^{*}$, Pichamol Jirapinyo, MD ${ }^{* 1,2}$, William Dong, BA ${ }^{*}$, \\ Marvin Ryou, MD ${ }^{* 1,2}$, Christopher C. Thompson, MD, MHES ${ }^{\star 1,2}$ \\ ${ }^{1 *}$ Developmental Endoscopy Lab, Harvard Medical School, Boston, MA \\ 2Division of Gastroenterology, Hepatology and Endoscopy, Brigham and Women's Hospital, \\ Boston, MA
}

\section{Abstract}

Objectives: Lumen-apposing metal stents (LAMS) are increasingly used for direct endoscopic necrosectomy (DEN) in the management of walled-off necrosis (WON). We compared LAMS and traditional cystoenterostomy in the management of WON.

\begin{abstract}
Methods: Retrospective analysis of patients who underwent DEN for management of WON. Primary outcome was rate of WON resolution. Secondary outcomes included technical and clinical success, time and number of procedures until resolution, requirement for alternative therapy, recurrence, and adverse events.
\end{abstract}

\begin{abstract}
Results: One hundred twelve patients underwent DEN with LAMS $(\mathrm{n}=34)$ or traditional cystoenterostomy $(\mathrm{n}=78)$. Mean WON size was $90.2 \times 60.1 \mathrm{~mm}$, and $61.8 \%$ had infected necrosis. Overall WON resolution was similar between LAMS and traditional cystoenterostomy (94.1\% vs $92.1 \%, P=0.510)$, with similar number of procedures until resolution (1.5 vs $1.5, P=$ 0.871 ). The LAMS were associated with faster resolution (86.9 vs 133.6 days, $P=0.038$ ), lower recurrence $(6.3 \%$ vs $23.1 \%, P=0.032)$, and decreased requirement for surgery $(0 \%$ vs $12.8 \%, P=$ $0.031)$ compared to traditional cystoenterostomy, but higher adverse event rates $(41.2 \%$ vs $7.7 \%, P$ $<0.001)$.
\end{abstract}

\footnotetext{
Address correspondence to: Christopher C. Thompson, MD, MHES, Developmental Endoscopy, Division of Gastroenterology, Hepatology and Endoscopy, Brigham and Women's Hospital, 75 Francis Street, Boston, MA 02115 (ccthompson@ bwh.harvard.edu). Tel: 617-525-8266, Fax: 617-264-6342.

DISCLOSURES

Phillip S. Ge has no conflicts of interest or financial ties to disclose. Joyce Y. Young has no conflicts of interest or financial ties to disclose.

Pichamol Jirapinyo has no conflicts of interest or financial ties to disclose.

William Dong has no conflicts of interest or financial ties to disclose.

Marvin Ryou: Medtronic (Consultant)

Christopher C. Thompson: Boston Scientific (Consultant), Medtronic (Consultant), USGI Medical (Consultant, Advisory Board Member, Research Support), Olympus (Consultant, Research Support), Apollo Endosurgery (Consultant, Research Support), GI Windows (Ownership Interest), Aspire Bariatrics (Research Support), Fractyl (Consultant, Advisory Board Member), Spatz (Research Support), EndoTAGSS (Ownership Interest), GI Dynamics (Consultant)

Presented in part at Digestive Diseases Week 2018, Washington, DC
} 
Conclusions: Despite higher adverse event rates, initial LAMS cystoenterostomy for management of WON results in faster resolution, lower recurrence, and decreased requirement for surgery.

\section{Keywords}

lumen apposing metal stent; LAMS; cystoenterostomy; necrosectomy; walled off necrosis; therapeutic endoscopic ultrasound

\section{INTRODUCTION}

Walled-off necrosis (WON) is defined as a mature encapsulated collection of pancreatic and/or peripancreatic necrosis characterized by a well-defined inflammatory wall, and differs from a pancreatic pseudocyst by the presence of solid necrotic material within the collection. ${ }^{1}$ The management of symptomatic WON has evolved substantially, from open surgical necrosectomy to minimally invasive approaches involving a combination of percutaneous catheter drainage and video-assisted retroperitoneal debridement. ${ }^{2}$ Endoscopic cystoenterostomy followed by endoscopic debridement (referred to as direct endoscopic necrosectomy; DEN) are minimally invasive alternatives that are increasingly becoming the standard of care for the management of symptomatic WON, and when feasible, are preferable to percutaneous and surgical approaches. ${ }^{3}$

The reported experience with endoscopic transluminal drainage has closely paralleled the evolution of endoscopic technology, including the advent of endoscopic ultrasound (EUS) and EUS-guided cystoenterostomy, the development of specialized stents from double pigtail (DP) plastic stents to fully-covered self-expanding metal stents (SEMS), and more recently, the introduction of lumen-apposing metal stents (LAMS). As such, the endoscopic experience and its associated data have been heterogeneous, and multiple endoscopic techniques have been described. ${ }^{4,5}$

Endoscopic ultrasound-guided cystoenterostomy with DEN is increasingly used for endoscopic management of WON. ${ }^{6-9}$ However, there is limited data directly comparing LAMS cystoenterostomy to traditional cystoenterostomy with DP drainage. In this study, we aimed to compare clinical outcomes and adverse events between LAMS cystoenterostomy and traditional cystoenterostomy with DP drainage in the endoscopic management of WON.

\section{MATERIALS AND METHODS}

\section{Patients}

Consecutive adult patients who underwent EUS-guided cystoenterostomy and direct endoscopic necrosectomy for walled off necrosis at our center were prospectively enrolled in a registry which was retrospectively analyzed for the purposes of this study. Institutional Review Board approval was obtained for this study.

Patients were included if they met the Revised Atlanta Classification for WON, had welldefined encapsulated collections with solid component on either computed tomography (CT), magnetic resonance imaging (MRI), or EUS, and underwent initial endoscopic 
management. Patients were excluded if they had pancreatic pseudocysts without evidence of WON, if they had other fluid collections that were endoscopically drained, or if they underwent initial percutaneous drainage or surgical necrosectomy.

\section{Endoscopic Procedures}

Following written informed consent, all patients were sedated under general endotracheal anesthesia. Broad-spectrum antibiotics were administered to all patients before and after the procedure. Walled-off necrosis was identified using a linear-array echoendoscope (GFUCT180; Olympus America, Center Valley, Pa) and carefully evaluated to assess for surrounding vasculature, and to ensure appropriateness for endoscopic cystoenterostomy.

All procedures were performed by endosonographers at our institution experienced in the endoscopic management of WON. At our institution, our practice for the endoscopic drainage of WON consisted of either traditional cystoenterostomy or LAMS cystoenterostomy based on endoscopist discretion and expertise. Transpapillary endoscopic treatment was not performed in addition to transmural drainage.

Traditional Cystoenterostomy With DP Drainage Technique-The WON was first punctured using a 19-gauge EUS needle, and fluid was aspirated and sent for bacterial cultures. Iodinated contrast was then injected into the WON to delineate the borders of the collection, assess for communication with the main pancreatic duct or other luminal structures, and to fully distend the wall of the collection to ease subsequent access. At our institution, the presence of a pancreatico-colonic or enteric fistula was considered a contraindication to endoscopic management, and thus contrast was routinely injected regardless of the cystoenterostomy technique. A standard 0.035 inch guidewire was then coiled into the WON under fluoroscopic guidance, and the EUS needle was exchanged over the wire using a Seldinger technique. Serial balloon dilation was performed up to $20 \mathrm{~mm}$ diameter over the guidewire, thus establishing the cystoenterostomy.

LAMS Cystoenterostomy Technique-Initial LAMS cystoenterostomy procedures were performed without electrocautery-assistance. The WON was punctured using a 19gauge EUS needle, and iodinated contrast was injected into the WON as described above. A 0.035 inch guidewire was then coiled into the WON under fluoroscopic guidance, and the EUS needle was exchanged over the wire using a Seldinger technique. Serial balloon dilation was performed, followed by deployment of a $10 \times 10 \mathrm{~mm}$ or $15 \times 10 \mathrm{~mm}$ LAMS (AXIOS; Boston Scientific, Marlborough, Mass) across the cystoenterostomy tract.

Following the advent of the electrocautery-enhanced LAMS, all subsequent procedures performed using the LAMS cystoenterostomy technique utilized a $10 \times 10 \mathrm{~mm}, 15 \times 10 \mathrm{~mm}$, or $20 \times 10 \mathrm{~mm}$ electrocautery-enhanced LAMS, deployed into the WON in a freehand fashion without the need for prior needle puncture or guidewire placement. Electrosurgical generator settings (ERBE USA, Inc, Marietta, Ga) included Auto Cut, 100 Watts, effect 5. Following LAMS placement, balloon dilation was performed across the LAMS, thus establishing the cystoenterostomy. 
Endoscopic Debridement Technique-After cystoenterostomy had been established, the WON was entered with a standard or therapeutic channel gastroscope. Endoscopic debridement was performed according to a standardized institutional protocol which consisted of limited fragmentation of densely necrotic material, followed by lavage of necrotic material with bacitracin 25,000 units in 1 liter of warmed saline. ${ }^{4}$

For patients who underwent the traditional cystoenterostomy technique, DP plastic stents (14 stents, with 5 French, 7 French, or 10 French diameter) were sequentially inserted across the cystoenterostomy tract following endoscopic debridement. For patients who underwent the LAMS cystoenterostomy technique, up to two additional DP plastic stents could be placed if deemed necessary at the endoscopist's discretion to secure the cystoenterostomy tract, prevent LAMS obstruction, and/or limit LAMS contact with the contralateral wall to reduce bleeding risk.

\section{Follow-Up}

Following EUS-guided cystoenterostomy and endoscopic debridement, all patients were scheduled for follow-up in a standardized fashion. In the absence of an adverse event requiring premature intervention, cross-sectional imaging, office visit, and endoscopic examination was performed every 4 weeks until resolution of the WON. If cross-sectional imaging demonstrated resolution of the WON, all stents were removed at follow-up endoscopy. If cross-sectional imaging demonstrated a persistent WON, repeat endoscopic debridement was performed. For patients who underwent traditional cystoenterostomy, the DP plastic stents were replaced following repeat endoscopic debridement. For patients who underwent LAMS cystoenterostomy, the LAMS was removed and DP plastic stents were placed following repeat endoscopic debridement. At the second intervention, the LAMS was always replaced with DP plastic stents in order to minimize the risk of bleeding from the metal flange of the LAMS coming into contact with the collapsing wall of the WON cavity. All proton pump inhibitors were discontinued until WON resolution. After WON resolution, patients were followed with repeat cross-sectional imaging 1-3 months following stent removal to assess for recurrence. Patients were additionally followed clinically for symptoms suspicious for WON recurrence, with imaging performed as indicated.

Unless already hospitalized, patients were admitted for observation following the procedure as clinically indicated. Bleeding was defined as clinically significant intraprocedural bleeding, delayed bleeding causing a drop of hemoglobin by $>2 \mathrm{~g} / \mathrm{dL}$, or requiring hospital admission, transfusion, or endoscopic intervention. Perforation was diagnosed either endoscopically or by the presence of extraluminal air seen on radiographic imaging. Mortality was defined as any procedure-related death prior to stent removal or within 60 days following stent removal.

\section{Study Outcomes}

The primary study outcome was the overall rate of WON resolution. Secondary outcomes included technical success; initial clinical success (defined as clinical success using the initial WON drainage modality); time to WON resolution; number of procedures until WON resolution; rate of failure to achieve WON resolution requiring alternative endoscopic 
therapy (i.e. failure of traditional cystoenterostomy requiring subsequent LAMS placement, and vice versa), percutaneous drainage, or surgical necrosectomy; WON recurrence following initial complete WON resolution; and adverse events.

\section{Data Analysis and Statistics}

Demographics were recorded including patient age, sex, and etiology of pancreatitis. Lesion characteristics were recorded including size of the WON collection, and presence of infected pancreatic necrosis. Procedural characteristics were recorded including the size, number, and type of stents used. Adverse events were recorded including bleeding, perforation or dislodgement during deployment, pneumoperitoneum, peritoneal leakage, abdominal pain requiring premature stent removal, stent occlusion or migration requiring premature procedure, and death related to complications from WON.

Descriptive statistics were used to characterize the demographics of the study population. Categorical data were summarized using counts and percentages, and continuous data were summarized using means and standard deviation (SD). Statistical analysis was performed using Fisher's exact test when comparing categorical variables, and Welch's $t$ test when comparing continuous variables. $P$ values of $<0.05$ were considered statistically significant.

\section{RESULTS}

\section{Demographics and WON Characteristics}

Demographics and WON characteristics are shown in Table 1. One hundred twelve consecutive patients underwent DEN during the study period, including 78 patients in the initial traditional cystoenterostomy with DP drainage group, and 32 patients in the initial LAMS cystoenterostomy group. The mean age was 52.1 (SD, 15.9) years and $38.4 \%$ patients were female. All patients were symptomatic, and $61.8 \%$ had evidence of infected pancreatic necrosis. The mean WON dimension was $90.2 \times 60.1 \mathrm{~mm}$ in cross-sectional diameter. Etiologies of pancreatitis included gallstone (29.7\%), alcohol (19.8\%), postpancreatectomy (11.7\%), hypertriglyceridemia $(7.2 \%)$, post-endoscopic retrograde cholangiopancreatography (ERCP) (6.3\%), medication-related (3.6\%), autoimmune (1.8\%), post-colonoscopy (0.9\%), and idiopathic (18.9\%). Walled-off necrosis due to pancreatectomy was more frequent in the LAMS cystoenterostomy compared to the traditional cystoenterostomy group $(23.5 \%$ vs $6.5 \%, P=0.014)$. In the initial LAMS cystoenterostomy group, 7 patients $(21.9 \%)$ had additional DP plastic stents placed within the LAMS to secure the cystoenterostomy tract.

\section{Clinical and Procedural Outcomes}

Clinical and procedural outcomes are shown in Table 2. The overall rate of WON resolution (primary outcome) was similar between the LAMS cystoenterostomy and traditional cystoenterostomy with DP drainage groups $(94.1 \%$ vs $92.1 \%, P=0.510)$.

Technical success was lower in the LAMS cystoenterostomy compared to the traditional cystoenterostomy group $(91.2 \%$ vs $100 \%, P=0.026)$. All patients who had a technical successful cystoenterostomy underwent successful DEN. Initial clinical success was similar 
between the LAMS and traditional cystoenterostomy groups ( $88.2 \%$ vs $76.9 \%, P=0.203)$. Time to WON resolution was shorter in the LAMS cystoenterostomy compared to the traditional cystoenterostomy group ( 86.9 vs 133.6 days, $P=0.038$ ). Both groups required similar numbers of total procedures until WON resolution ( 1.5 vs 1.5 procedures, $P=$ $0.871)$.

Among patients who had failed to achieve WON resolution following initial traditional cystoenterostomy, 4 patients $(5.1 \%)$ subsequently underwent successful LAMS placement with WON resolution, 5 patients (6.4\%) required percutaneous drainage, and 10 patients (12.8\%) required surgical necrosectomy. Among patients who had failed to achieve WON resolution following initial LAMS cystoenterostomy, 4 patients (11.8\%) subsequently underwent successful DP plastic stent placement with WON resolution, and 1 patient (2.9\%) required percutaneous drainage. No patients who underwent initial LAMS cystoenterostomy required surgical necrosectomy $(P=0.031)$. Recurrence rates of WON (following initial complete resolution) were significantly lower in the LAMS cystoenterostomy group compared to the traditional cystoenterostomy group $(6.3 \%$ vs $24.1 \%, P=0.032)$.

\section{Adverse Events}

Adverse events are shown in Table 3. Adverse events were significantly higher in the LAMS cystoenterostomy group when compared to the traditional cystoenterostomy group (41.2\% vs $7.7 \%, P<0.001)$.

There were three patients who had a perforation or dislodgement during LAMS cystoenterostomy. In each case, the LAMS was immediately retrieved and the perforation was closed successfully using endoscopic clips. Following endoscopic closure, a second cystoenterostomy was successfully created at a different location with LAMS in two cases and with DP plastic stents in one case.

There were four patients who experienced clinically significant bleeding. One patient in the traditional cystoenterostomy group experienced refractory intraprocedural bleeding at the cystoenterostomy site which required emergent angiography and embolization. Three patients in the LAMS cystoenterostomy group experienced postprocedural bleeding. One patient developed melena 6 weeks following LAMS placement in the context of therapeutic anticoagulation and was found to have a bleeding ulcer on the gastric wall opposite of the LAMS, which was endoscopically treated. Another patient developed syncope 6 weeks following LAMS placement and was found to have a bleeding pseudoaneurysm within the WON cavity which required emergent angiography and embolization. A third patient developed severe acute epigastric abdominal pain one day following LAMS placement and was found to have self-limited bleeding into the WON cavity which resolved with conservative management without need for transfusion.

Other adverse events among patients who underwent initial LAMS cystoenterostomy included one patient who experienced severe abdominal pain requiring premature LAMS removal, four patients with LAMS occlusion requiring premature endoscopic intervention, one patient with LAMS migration requiring premature endoscopic intervention, one patient with development of unexplained pneumoperitoneum following LAMS placement, and one 
patient who died from complications from unresolved WON. Other adverse events among patients who underwent initial traditional cystoenterostomy included two patients with DP stent occlusion requiring premature endoscopic intervention, one patient with DP stent migration requiring premature endoscopic intervention, one patient with development of unexplained pneumoperitoneum, and one patient with development of a peritoneal leak.

\section{DISCUSSION}

Since its introduction, EUS-guided drainage has increasingly become adopted as the procedure of choice for symptomatic mature pancreatic fluid collections. ${ }^{6-12}$ Despite high clinical success rates using the traditional cystoenterostomy technique for pseudocyst drainage, clinical success was lower for drainage of WON due to stent occlusion and superimposed infection which was believed to be primarily due to the need for endoscopic debridement. ${ }^{13,14}$ Additional approaches such as the multiple transluminal gateway technique were developed to address this issue. ${ }^{5}$ Covered esophageal and biliary SEMS were also utilized and allowed for more efficient drainage; however, their straight design resulted in a high rate of stent migration and tissue injury from stent erosion into either the wall of the WON collection or gastrointestinal tract. ${ }^{15}$

The electrocautery-enhanced LAMS is a dedicated device designed and approved for EUSguided drainage of pancreatic fluid collections such as pseudocysts and WON. First introduced in 2014, early experience with LAMS for drainage of pancreatic fluid collections showed high overall technical success (89-100\%) and clinical success (93-100\%) rates; however, notable adverse events have been reported including stent misdeployment, perforation, bleeding, and stent occlusion typically due to ingested food or necrotic pancreatic debris. ${ }^{16-23}$ Due to its simplified deployment and ease of use, LAMS has become the preferred stent for endoscopic drainage of pancreatic fluid collections.

Studies directly comparing traditional cystoenterostomy with DP drainage with LAMS cystoenterostomy have yielded mixed results and have been hampered by their heterogeneity and comparison of multiple disparate techniques. Siddiqui et al compared patients who underwent DEN with either DP plastic stents, SEMS, or LAMS. ${ }^{24}$ Although technical success was similar between the 3 groups, the SEMS group was found to have the highest rate of WON resolution and lowest rate of early adverse events. The LAMS group had the lowest mean number of procedures required for WON resolution, whereas the DP group had the highest. Abu Dayyeh et al compared patients who underwent DEN with either DP plastic stents or metal stents (which included both SEMS and LAMS). ${ }^{25}$ They showed that while the techniques similarly effective, metal stents were associated with decreased need for repeated procedures $(60.4 \%$ vs $30.8 \%, P=0.01)$ and decreased risk for clinically significant bleeding ( $14 \%$ vs $2 \%, P=0.02)$. Lang et al demonstrated similar efficacy between DP plastic stents and LAMS; however, LAMS was instead associated with higher rates of procedure-related bleeding ( $19 \%$ vs $1 \%, P=0.0003)$ and greater need for repeat endoscopic intervention (26\% vs $10 \%, P=0.07) .{ }^{26}$ Notably, there was significant heterogeneity with only $17 \%$ of patients in the DP plastic stent group having WON, compared to $47 \%$ in the LAMS group. 
In order to address these mixed results, Bazerbachi et al recently reported a meta-analysis comparing patients who underwent DEN with either DP plastic stents or metal stents (which included both SEMS and LAMS). Their results suggested that metal stents were superior to DP plastic stents for WON resolution, with fewer bleeding events and a trend towards lower stent occlusion and perforation rate, but with increased stent migration rate. ${ }^{27}$ However, the meta-analysis was likewise hampered by heterogeneity between the included studies, and the combination of both LAMS and SEMS in the analysis limited the ability to derive conclusions regarding either stent.

In this study, we directly compared two specific DEN techniques (traditional cystoenterostomy with DP drainage versus initial LAMS cystoenterostomy, with similar degree and method of endoscopic debridement) for management of WON with minimal variability in technique. We were able to demonstrate similar success rates for WON resolution between both LAMS and traditional cystoenterostomy groups $(94.1 \%$ vs $92.1 \%, P$ $=0.510$ ). Both groups required similar numbers of total procedures until WON resolution (1.5 vs 1.5 procedures, $P=0.871$ ). We found that patients who underwent initial LAMS cystoenterostomy had significantly decreased time to resolution (86.9 vs 133.6 days, $P=$ $0.038)$ and lower rates of WON recurrence $(6.3 \%$ vs $23.1 \%, P=0.032)$ when compared to traditional cystoenterostomy, likely due to the larger caliber of the LAMS and more efficient drainage of necrotic contents as compared to DP plastic stents. Furthermore, patients who underwent LAMS cystoenterostomy had a decreased requirement for surgical necrosectomy when compared to traditional cystoenterostomy ( $0 \%$ vs $12.8 \%, P=0.031$ ). However, LAMS cystoenterostomy was associated with significantly increased adverse event rates when compared to patients who underwent initial traditional cystoenterostomy $(41.2 \%$ vs $7.7 \%, P$ $<0.001)$, particularly with respect to bleeding $(P=0.083)$, stent occlusion $(P=0.068)$, and perforation/dislodgement during deployment $(P=0.026)$. Nonetheless, we have continued to utilize LAMS cystoenterostomy and believe that its increase in adverse events is offset by its superior clinical outcomes when compared to traditional cystoenterostomy and can be mitigated through further refinements in technique.

Given the complexity and inherent risks of EUS-guided cystoenterostomy and endoscopic debridement, all steps in DEN have been standardized in our institution for each of the two endoscopic treatment options. In the absence of an adverse event requiring premature intervention, all patients were followed up at 4 week intervals until resolution of the WON. Following early experience with delayed postprocedural bleeding in patients undergoing LAMS cystoenterostomy, it has been our standard practice to remove the LAMS within 4 weeks of stent placement in order to minimize bleeding complications from the metal flange of the LAMS coming into contact with the collapsing wall of the WON cavity. If the WON cavity had not resolved at 4 weeks following initial cystoenterostomy, the LAMS was always removed and replaced with DP plastic stents for the same reason.

During initial LAMS placement, careful attention is also paid to the presence of any splenic artery pseudoaneurysms or other prominent vasculature particularly on the contralateral wall; if present, DP plastic stents are placed across the LAMS to reduce the risk of bleeding from the metal flange of the LAMS coming into contact with these vessels. In addition, to avoid early stent occlusion, DP plastic stents are placed across the LAMS if the WON cavity 
is characterized by dense necrosis. Finally, in order to facilitate resolution, DP plastic stents are also placed across the LAMS if the WON cavity is particularly large, irregular in shape, or multilocular in morphology.

There are notable limitations to our study including its retrospective nature. Our study was performed at a major tertiary care academic hospital, and procedures were performed by endosonographers highly experienced in the management of WON, which may limit the applicability of our findings. However, we believe that our findings would likely be amplified for less-experienced endoscopists, especially as LAMS becomes increasingly popular outside academic hospital settings. In addition, a minority of patients in the LAMS cystoenterostomy group (21.9\%) also had concurrent DP plastic stents placed to stabilize the cystoenterostomy tract; while this may have introduced bias into the study, we do not believe this would have significantly impacted our results. Finally, cystoenterostomy techniques have changed over time and with increasing experience, which may have introduced additional bias into the study.

In conclusion, initial LAMS cystoenterostomy for endoscopic management of WON results in significantly decreased time to resolution, lower rates of recurrence, and lower requirement for surgical necrosectomy when compared to traditional cystoenterostomy with DP drainage. However, initial LAMS cystoenterostomy is associated with increased adverse event rates when compared to traditional cystoenterostomy, which may be mitigated with early surveillance imaging, expedient LAMS removal, and placement of DP plastic stents within the LAMS when there dense necrosis or adjacent vasculature is present. Further prospective studies will seek to better delineate the optimal initial stent choice and drainage procedure for symptomatic WON.

\section{Acknowledgments}

Funding Support: None

\section{REFERENCES}

1. Banks PA, Bollen TL, Dervenis C, et al. Classification of acute pancreatitis--2012: revision of the Atlanta classification and definitions by international consensus. Gut. 2013;62:102-111. [PubMed: 23100216]

2. van Santvoort HC, Besselink MG, Bakker OJ, et al. A step-up approach or open necrosectomy for necrotizing pancreatitis. N Engl J Med. 2010;362:1491-1502. [PubMed: 20410514]

3. Ge PS, Weizmann M, Watson RR. Pancreatic Pseudocysts: Advances in Endoscopic Management. Gastroenterol Clin North Am. 2016;45:9-27. [PubMed: 26895678]

4. Thompson CC, Kumar N, Slattery J, et al. A standardized method for endoscopic necrosectomy improves complication and mortality rates. Pancreatology. 2016;16:66-72. [PubMed: 26748428]

5. Varadarajulu S, Phadnis MA, Christein JD, et al. Multiple transluminal gateway technique for EUSguided drainage of symptomatic walled-off pancreatic necrosis. Gastrointest Endosc. 2011;74:7480. [PubMed: 21612778]

6. Kumar N, Conwell DL, Thompson CC. Direct endoscopic necrosectomy versus step-up approach for walled-off pancreatic necrosis: comparison of clinical outcome and health care utilization. Pancreas. 2014;43:1334-1339. [PubMed: 25083997]

7. Bakker OJ, van Santvoort HC, van Brunschot S, et al. Endoscopic transgastric vs surgical necrosectomy for infected necrotizing pancreatitis: a randomized trial. JAMA. 2012;307:10531061. [PubMed: 22416101] 
8. van Brunschot S, van Grinsven J, van Santvoort HC, et al. Endoscopic or surgical step-up approach for infected necrotising pancreatitis: a multicentre randomised trial. Lancet. 2018;391:51-58. [PubMed: 29108721]

9. Khan MA, Kahaleh M, Khan Z, et al. Time for a Changing of Guard: From Minimally Invasive Surgery to Endoscopic Drainage for Management of Pancreatic Walled-off Necrosis. J Clin Gastroenterol. 2019;53:81-88. [PubMed: 30383567]

10. Sharaiha RZ, DeFilippis EM, Kedia P, et al. Metal versus plastic for pancreatic pseudocyst drainage: clinical outcomes and success. Gastrointest Endosc. 2015;82:822-827. [PubMed: 25936453]

11. Gluck M, Ross A, Irani S, et al. Endoscopic and percutaneous drainage of symptomatic walled-off pancreatic necrosis reduces hospital stay and radiographic resources. Clin Gastroenterol Hepatol. 2010;8:1083-1088. [PubMed: 20870036]

12. Varadarajulu S, Wilcox CM, Latif S, et al. Management of pancreatic fluid collections: a changing of the guard from surgery to endoscopy. Am Surg. 2011;77:1650-1655. [PubMed: 22273224]

13. Baron TH, Harewood GC, Morgan DE, et al. Outcome differences after endoscopic drainage of pancreatic necrosis, acute pancreatic pseudocysts, and chronic pancreatic pseudocysts. Gastrointest Endosc. 2002;56:7-17. [PubMed: 12085029]

14. Hookey LC, Debroux S, Delhaye M, et al. Endoscopic drainage of pancreatic-fluid collections in 116 patients: a comparison of etiologies, drainage techniques, and outcomes. Gastrointest Endosc. 2006;63:635-643. [PubMed: 16564865]

15. Talreja JP, Shami VM, Ku J, et al. Transenteric drainage of pancreatic-fluid collections with fully covered self-expanding metallic stents (with video). Gastrointest Endosc. 2008;68:1199-1203. [PubMed: 19028232]

16. Itoi T, Binmoeller KF, Shah J, et al. Clinical evaluation of a novel lumen-apposing metal stent for endosonography-guided pancreatic pseudocyst and gallbladder drainage (with videos). Gastrointest Endosc. 2012;75:870-876. [PubMed: 22301347]

17. Rinninella E, Kunda R, Dollhopf M, et al. EUS-guided drainage of pancreatic fluid collections using a novel lumen-apposing metal stent on an electrocautery-enhanced delivery system: a large retrospective study (with video). Gastrointest Endosc. 2015;82:1039-1046. [PubMed: 26014960]

18. Shah RJ, Shah JN, Waxman I, et al. Safety and efficacy of endoscopic ultrasound-guided drainage of pancreatic fluid collections with lumen-apposing covered self-expanding metal stents. Clin Gastroenterol Hepatol. 2015;13:747-752. [PubMed: 25290534]

19. Walter D, Will U, Sanchez-Yague A, et al. A novel lumen-apposing metal stent for endoscopic ultrasound-guided drainage of pancreatic fluid collections: a prospective cohort study. Endoscopy. 2015;47:63-67. [PubMed: 25268308]

20. Siddiqui AA, Adler DG, Nieto J, et al. EUS-guided drainage of peripancreatic fluid collections and necrosis by using a novel lumen-apposing stent: a large retrospective, multicenter U.S. experience (with videos). Gastrointest Endosc. 2016;83:699-707. [PubMed: 26515956]

21. Vazquez-Sequeiros E, Baron TH, Perez-Miranda M, et al. Evaluation of the short- and long-term effectiveness and safety of fully covered self-expandable metal stents for drainage of pancreatic fluid collections: results of a Spanish nationwide registry. Gastrointest Endosc. 2016;84:450-457 e452. [PubMed: 26970012]

22. Sharaiha RZ, Tyberg A, Khashab MA, et al. Endoscopic Therapy With Lumen-apposing Metal Stents Is Safe and Effective for Patients With Pancreatic Walled-off Necrosis. Clin Gastroenterol Hepatol. 2016;14:1797-1803. [PubMed: 27189914]

23. Gornals JB, De la Serna-Higuera C, Sanchez-Yague A, et al. Endosonography-guided drainage of pancreatic fluid collections with a novel lumen-apposing stent. Surg Endosc. 2013;27:1428-1434. [PubMed: 23232994]

24. Siddiqui AA, Kowalski TE, Loren DE, et al. Fully covered self-expanding metal stents versus lumen-apposing fully covered self-expanding metal stent versus plastic stents for endoscopic drainage of pancreatic walled-off necrosis: clinical outcomes and success. Gastrointest Endosc. 2017;85:758-765. [PubMed: 27566053] 
25. Abu Dayyeh BK, Mukewar S, Majumder S, et al. Large-caliber metal stents versus plastic stents for the management of pancreatic walled-off necrosis. Gastrointest Endosc. 2018;87:141-149. [PubMed: 28478030]

26. Lang GD, Fritz C, Bhat T, et al. EUS-guided drainage of peripancreatic fluid collections with lumen-apposing metal stents and plastic double-pigtail stents: comparison of efficacy and adverse event rates. Gastrointest Endosc. 2018;87:150-157. [PubMed: 28713067]

27. Bazerbachi F, Sawas T, Vargas EJ, et al. Metal stents versus plastic stents for the management of pancreatic walled-off necrosis: a systematic review and meta-analysis. Gastrointest Endosc. 2018;87:30-42.e15. [PubMed: 28867073] 
TABLE 1.

Demographics and WON Characteristics

\begin{tabular}{lccc}
\hline & LAMS, $\mathbf{n}(\boldsymbol{\%})$ & Traditional, $\mathbf{n}(\boldsymbol{\%})$ & $\boldsymbol{P}$ \\
\hline Demographics & & & \\
$\quad$ Total patients & $34(30.4)$ & $78(69.6)$ & - \\
Age, mean (SD), y & $52.5(17.4)$ & $51.9(15.3)$ & 0.857 \\
$\quad$ Sex, female & $13(38.2)$ & $30(38.5)$ & 1.000 \\
Etiology of WON & & & \\
Alcohol & $4(11.8)$ & $18(23.4)$ & 0.203 \\
Gallstone & $8(23.5)$ & $25(32.5)$ & 0.381 \\
Hypertriglyceridemia & $3(8.8)$ & $5(6.5)$ & 0.697 \\
Medication & $1(2.9)$ & $3(3.9)$ & 1.000 \\
Autoimmune & $1(2.9)$ & $1(1.3)$ & 1.000 \\
Idiopathic & $7(20.6)$ & $14(18.2)$ & 0.794 \\
Post-pancreatectomy & $8(23.5)$ & $5(6.5)$ & $\mathbf{0 . 0 1 4}$ \\
Post-ERCP & $1(2.9)$ & $6(7.8)$ & 0.437 \\
Post-colonoscopy & $1(2.9)$ & $0(0.0)$ & 0.304 \\
WON Characteristics & & & \\
Long dimension, mean (SD), mm & $84.7(45.9)$ & $92.7(46.2)$ & 0.403 \\
Short dimension, mean (SD), mm & $58.8(22.9)$ & $60.7(33.2)$ & 0.745 \\
Presence of infection & $19(57.6)$ & $49(63.6)$ & 0.532 \\
\hline
\end{tabular}

Bold values denote statistical significance.

ERCP, endoscopic retrograde cholangiopancreatography; LAMS, lumen apposing metal stent cystoenterostomy; SD, standard deviation; Traditional, traditional cystoenterostomy with DP drainage; WON, walled off necrosis. 
TABLE 2.

\section{Clinical Outcomes}

\begin{tabular}{lccc}
\hline & LAMS, n (\%) & Traditional, n (\%) & $\boldsymbol{P}$ \\
\hline Technical success & $31(91.2)$ & $78(100.0)$ & $\mathbf{0 . 0 2 6}$ \\
Initial clinical success & $30(88.2)$ & $60(76.9)$ & 0.203 \\
Resolution of WON & $32(94.1)$ & $70(92.1)$ & 0.510 \\
Time to resolution, mean (SD), d & $86.9(85.1)$ & $133.6(136.5)$ & $\mathbf{0 . 0 3 8}$ \\
Number of procedures until resolution, mean (SD) & $1.5(0.8)$ & $1.5(0.8)$ & 0.871 \\
Recurrence of WON & $2(6.3)$ & $18(23.1)$ & $\mathbf{0 . 0 3 2}$ \\
Switched from traditional to LAMS cystoenterostomy & - & $4(5.1)$ & - \\
Switched from LAMS to traditional cystoenterostomy & $4(11.8)$ & - & - \\
Required percutaneous drainage & $1(2.9)$ & $5(6.4)$ & 0.666 \\
Required surgical necrosectomy & $0(0.0)$ & $10(12.8)$ & $\mathbf{0 . 0 3 1}$ \\
\hline
\end{tabular}

Bold values denote statistical significance.

LAMS indicates lumen apposing metal stent cystoenterostomy; SD, standard deviation; Traditional, traditional cystoenterostomy with DP drainage; WON, walled off necrosis. 
TABLE 3.

\begin{tabular}{|c|c|c|c|}
\hline & LAMS, n (\%) & Traditional, n (\%) & $P$ \\
\hline Total adverse events & $14(41.2)$ & $6(7.7)$ & $<0.001$ \\
\hline Pain requiring premature stent removal & $1(2.9)$ & $0(0.0)$ & 0.304 \\
\hline Bleeding & $3(8.8)$ & $1(1.3)$ & 0.083 \\
\hline Stent occlusion requiring premature procedure & $4(11.8)$ & $2(2.6)$ & 0.068 \\
\hline Stent migration requiring premature procedure & $1(2.9)$ & $1(1.3)$ & 1.000 \\
\hline Perforation/dislodgement during deployment & $3(8.8)$ & $0(0.0)$ & 0.026 \\
\hline Pneumoperitoneum & $1(2.9)$ & $1(1.3)$ & 1.000 \\
\hline Peritoneal leak & $0(0.0)$ & $1(1.3)$ & 1.000 \\
\hline Death prior to WON resolution & $1(2.9)$ & $0(0.0)$ & 0.304 \\
\hline
\end{tabular}

Bold values denote statistical significance.

LAMS indicates lumen apposing metal stent cystoenterostomy; Traditional, traditional cystoenterostomy with DP drainage; WON, walled off necrosis. 